\title{
DECLÍNIO COGNITIVO: CONHECIMENTOS, ATITUDES E PRÁTICAS PREVENTIVAS DE IDOSOS EM COMUNIDADE*
}

\author{
Priscila Aguiar Mendes ${ }^{1}$, Annelita Almeida Oliveira Reiners², Rosemeiry Capriata de Souza Azevedo ${ }^{3}$, \\ Ana Carolina Macri Gaspar ${ }^{1}$, Neuber José Segri ${ }^{4}$
}

Objetivo: avaliar o conhecimento, as atitudes e as práticas preventivas do declínio cognitivo realizadas pelos idosos. Método: estudo transversal, desenvolvido com 557 idosos atendidos nas Unidades de Saúde da Família de um município de Mato Grosso, nos meses de fevereiro a maio de 2015. Os dados foram coletados por meio de entrevista, utilizando questionário estruturado com perguntas sobre conhecimentos, atitudes e práticas preventivas de declínio cognitivo baseado nos inquéritos: Conhecimento, Atitude e Práticas. Realizou-se análise bivariada entre as variáveis conhecimento, atitude e práticas preventivas de declínio cognitivo e regressão múltipla de Poisson pelo método stepwise forward. Resultados: os idosos com conhecimento insatisfatório foram 469 (84,2\%); atitude favorável, 523 (93,9\%); e desenvolvem práticas preventivas do declínio cognitivo, 307 (55,1\%). Foi encontrada associação entre conhecimento satisfatório e práticas preventivas de declínio cognitivo $(p=0,027)$. Conclusão: Tais achados fornecem subsídios para ações em saúde, com a finalidade de elevar a realização das práticas preventivas nessa população.

DESCRITORES: Cognição; Conhecimentos, atitudes e prática em saúde; Idoso; Enfermagem geriátrica; Prevenção de doenças.

\section{COGNITIVE DECLINE: KNOWLEDGE, ATTITUDES AND PREVENTIVE PRACTICES OF OLDER ADULTS IN THE COMMUNITY}

Objective: to evaluate the knowledge, attitudes and practices for the prevention of cognitive decline carried out by older adults. Method: a crosssectional study was carried out with 557 older adults attended at the Family Health Units of a municipality of Mato Grosso from February to May of 2015. The data were collected through an interview using a structured questionnaire with questions on knowledge, attitudes and practices for the prevention of cognitive decline based on Knowledge, Attitude and Practice surveys. A bivariate analysis was performed between the variables knowledge, attitudes and practices for the prevention of cognitive decline, as well as Poisson multiple regression by the forward stepwise method. Results: $469(84.2 \%)$ of the older adults presented unsatisfactory knowledge; $523(93.9 \%)$ a favorable attitude; and 307 (55.1\%) performed practices to prevent cognitive decline. An association was found between satisfactory knowledge and practices for the prevention of cognitive decline ( $p=0.027)$. Conclusion: These findings provide support for health actions that aim to increase the performance of preventive practices in this population. KEYWORDS: Cognition; Knowledge, attitudes and practice in health; Older adult; Geriatric nursing; Disease prevention.

\section{DECLIVE COGNITIVO: CONOCIMIENTOS, ACTITUDES Y PRÁCTICAS PREVENTIVAS DE ANCIANOS EN COMUNIDAD}

\begin{abstract}
Objetivo: evaluar el conocimiento, las actitudes y las prácticas preventivas del declive cognitivo que ocurre en ancianos. Método: estudio trasversal, desarrollado con 557 ancianos atendidos en las Unidades de Salud de la Familia de un municipio de Mato Grosso, en los meses de febrero a mayo de 2015. Se recogieron los datos por medio de entrevista, utilizándose cuestionario estructurado con preguntas acerca de conocimientos, actitudes y prácticas preventivas de declive cognitivo considerándose las investigaciones: Conocimiento, Actitud y Prácticas. Se realizó análisis bivariado entre las variables conocimiento, actitud y prácticas preventivas de declive cognitivo y regresión múltiple de Poisson por el método stepwise forward. Resultados: Ios ancianos con conocimiento insatisfactorio fueron 469 (84,2\%); actitud favorable, 523 (93,9\%); y desarrollan prácticas preventivas del declive cognitivo, 307 (55,1\%). Hubo asociación entre conocimiento satisfactorio y prácticas preventivas de declive cognitivo ( $\mathrm{p}=0$,027). Conclusión: Esos hallazgos dan subsidios para acciones en salud y pueden elevar la realización de las prácticas preventivas en esa población.

DESCRIPTORES: Cognición; Conocimientos, actitudes y práctica en salud; Anciano; Enfermería geriátrica; Prevención de enfermedades.
\end{abstract}

"Extraído da dissertação "PRÁTICAS PREVENTIVAS DE DECLíNIO COGNITIVO REALIZADAS POR IDOSOS E FATORES ASSOCIADOS", Programa de Pós-graduação em Enfermagem - Mestrado em Enfermagem da Faculdade de Enfermagem da Universidade Federal de Mato Grosso, Brasil, 2016.

${ }^{1}$ Enfermeira. Mestre em Enfermagem pelo Programa de Pós-graduação da Faculdade de Enfermagem da Universidade Federal de Mato Grosso, campus Cuiabá. Docente no Curso de Graduação em Enfermagem da Universidade do Estado de Mato Grosso. Tangará da Serra, MT, Brasil.

Enfermeira. Doutora em Enfermagem. Docente da Faculdade de Enfermagem da Universidade Federal de Mato Grosso. Cuiabá, MT, Brasil.

${ }^{3}$ Enfermeira. Doutora em Enfermagem. Docente da Faculdade de Enfermagem da Universidade Federal de Mato Grosso. Cuiabá, MT, Brasil.

${ }^{4}$ Estatístico. Doutor em Ciências. Docente na Universidade Federal de Mato Grosso. Cuiabá, MT, Brasil.

Autor Correspondente:

Priscila Aguiar Mendes.

Recebido: $21 / 09 / 2017$

Universidade Estadual de Mato Grosso (UNEMAT).

Finalizado: $17 / 04 / 2018$

Rua 10 A, no 411 W - 78300-000. Tangará da Serra, Mato Grosso, Brasil.

E-mail: prih.mendes@gmail.com. 


\section{INTRODUÇÃO}

A demência é uma síndrome de natureza crônica e progressiva, caracterizada por comprometimento das funções cognitivas, como memória, pensamento e comportamento, que prejudica a realização das Atividades de Vida Diária (AVD). ${ }^{(1)}$ Essa síndrome afeta tanto os idosos quanto os seus familiares de maneira pessoal, emocional, social e econômica, e, dos casos de demência, a Doença de Alzheimer (DA) é o subtipo demencial mais frequente $(50 \% \text { a } 75 \%)^{.2)}$

A demência possui elevada prevalência na população mundial e afeta principalmente as pessoas mais velhas, com aumento da probabilidade de desenvolvê-la após os 65 anos. ${ }^{(2)} \mathrm{Em} \mathrm{2010,} \mathrm{35,6} \mathrm{milhões}$ de pessoas tinham esta condição, e estima-se que haja duplicação dos casos a cada 20 anos, chegando a 115,4 milhões de pessoas com demência no ano de 2050. Em países em desenvolvimento e em transição demográfica como o Brasil espera-se um aumento nos casos de demência maior que a média mundial. ${ }^{(1)}$

No ano de 2010, foram gastos muitos recursos com a assistência médica, social e cuidado informal em pacientes com demência. A soma dos valores totais estimado foi de 604 bilhões de dólares em todo o mundo ${ }^{(2)}$ e no Brasil 7.209 bilhões de dólares. ${ }^{(3)}$

O Declínio Cognitivo (DC) pode decorrer de alterações fisiológicas esperadas durante o processo de envelhecimento; entretanto, alterações de diversos graus que afetam a cognição, maiores que o esperado para a idade e escolaridade, podem ser o estágio de transição para as demências. ${ }^{(4)} \mathrm{O}$ Comprometimento Cognitivo Leve $(\mathrm{CCL})$ é considerado a fase intermediária entre as alterações do DC - decorrentes do envelhecimento normal - e a demência, e pode ter seu início sem comprometer a autonomia e a independência do idoso ou ainda evoluir para a demência com incapacidade funcional total. ${ }^{(5)}$

Evidências científicas recentes indicam que determinadas práticas podem postergar o DC e reduzir o risco de desenvolvimento da demência. Atividade física, dieta do mediterrâneo, interação social, controle de riscos cardiovasculares - hipertensão arterial, diabetes e hipercolesterolemia, e estimulação cognitiva, por meio da leitura, jogos de tabuleiro, palavras cruzadas e uso do computador, por exemplo, são práticas recomendadas para a prevenção do DC. ${ }^{(2,6)}$

Alguns fatores têm sido associados às práticas preventivas de DC, como sexo, idade, escolaridade, estado civil, renda, autopercepção de saúde, capacidade física e mental, humor e motivação, tipo, duração e intensidade das atividades, interação social e apoio da família. ${ }^{(7-9)}$ Fatores como a falta e/ou imprecisão de conhecimento e crenças equivocadas sobre o DC são um problema global e preocupam as entidades governamentais e não governamentais. ${ }^{(2,10)}$ Tais fatores podem gerar medo, sensação de insegurança, provocar estigmas e isolamento social, ocasionar atrasos na detecção, tratamento e cuidados da doença, assim como influenciar a prática e o engajamento das pessoas na prevenção do DC. ${ }^{(1,11)}$

Nessa perspectiva, é crescente o interesse de estudiosos em investigar conhecimentos, percepções, estigmas e crenças em relação ao DC, particularmente a $\mathrm{DA}^{(10,12)}$, com o argumento teórico de que a maneira como as pessoas pensam e interpretam as coisas determina as suas respostas emocionais e comportamentais. ${ }^{(11)}$ Neste sentido, torna-se importante o desenvolvimento de estudos com esta temática, no sentido de contribuir para o conhecimento deste fenômeno, bem como auxiliar no planejamento de intervenções eficazes, a fim de promover a saúde cognitiva da população idosa.

O objetivo deste estudo foi avaliar o conhecimento, as atitudes e as práticas preventivas do declínio cognitivo realizadas pelos idosos.

\section{MÉTODO}

Estudo quantitativo, transversal e analítico, desenvolvido no município de Tangará da Serra - MT, nos meses de fevereiro a maio de 2015. 
Participaram da pesquisa idosos residentes no perímetro urbano do município, atendidos nas Unidades de Saúde da Família (USF) registradas no Cadastro Nacional de Estabelecimentos de Saúde (CNES). Após levantamento nas fichas e prontuários do número de idosos atendidos em cada USF, a amostra probabilística estratificada proporcional foi calculada por meio da fórmula para populações finitas, com margem de erro de 4\%, prevalência do evento de 50\% e nível de confiança de 95\%, totalizando 557 participantes.

Essa amostra foi dividida em 10 estratos, cada um deles representado por uma USF. O número de idosos que deveriam participar da pesquisa foi dividido por estrato, calculado de forma proporcional, com base na representatividade de cada USF em relação à população do estudo. Após o cálculo, os participantes de cada estrato foram selecionados por meio de amostra aleatória simples.

Os critérios de inclusão foram: apresentar capacidade de comunicação e funções cognitivas preservadas, avaliadas pelo Mini Exame do Estado Mental (MEEM). O ponto de corte adotado foi de acordo com a escolaridade, uma vez que esse fator interfere no desempenho do teste: analfabetos $=19$ pontos; 1 a 3 anos de escolaridade $=23$ pontos; 4 a 7 anos de escolaridade $=24$ pontos; e $>7$ anos de escolaridade $=28$ pontos. ${ }^{(13)}$

Um novo idoso foi sorteado nos casos de não atendimento ao critério de inclusão: óbitos e após não ter sido encontrado em seu domicílio, por três vezes consecutivas, em horários e dias distintos. Além desses casos, durante a coleta de dados, 116 idosos apresentaram alterações cognitivas após aplicação do MEEM e por isso não foram incluídos na pesquisa. .

Os dados foram coletados por meio de entrevista na residência do participante, utilizando um questionário formulado pela pesquisadora, contendo questões referentes às variáveis sociodemográficas e às condições de saúde. As questões sobre conhecimento, atitude e práticas preventivas de DC realizadas por idosos foram elaboradas a partir de modelos existentes em inquéritos CAP (Conhecimento, Atitudes e Práticas) ${ }^{(14)}$ e seguiu-se as recomendações contidas na literatura sobre o assunto. ${ }^{(2,6)}$ Foi realizado um teste piloto em janeiro de 2015, com 14 idosos em Cuiabá - MT e o questionário foi ajustado antes da coleta de dados, realizada pela pesquisadora principal e uma mestranda devidamente capacitada.

As variáveis sociodemográficas são sexo, idade, estado civil, ocupação, renda e escolaridade. As de condições de saúde são problemas de saúde, uso de medicamentos e autoavaliação de saúde.

O conhecimento foi definido como os saberes dos idosos referentes ao $\mathrm{DC}^{(15)} \mathrm{e}$ foi avaliado por meio de 17 questões sobre DC e práticas de prevenção, com opções de resposta sim/não/não sei e apenas uma alternativa correta valendo 1 ponto. O escore do conhecimento variou de 0 a 17 pontos. Com base no acerto de $50 \%$ das questões mais uma, o conhecimento foi considerado satisfatório para aqueles que obtiveram escore maior ou igual a 10 pontos e insatisfatório para aqueles que pontuaram até 9 pontos.

A atitude, definida a partir do conceito apresentado por um estudo do tipo $\mathrm{CAP}^{(16)}$ compreende as opiniões, crenças e predisposições que os idosos têm em relação ao DC, e avaliada por meio de 19 questões, com opções de resposta sim/não e apenas 1 alternativa correta. A pontuação variou de 0 a 19 pontos. Foi considerado como atitude favorável o acerto de $50 \%$ das questões mais uma, ou seja, o participante que obteve escore maior ou igual a 11 pontos e não favorável para aqueles com até 10 pontos.

A variável dependente - práticas preventivas de DC - foi verificada por meio do autorrelato e definida como as várias atividades realizadas pelos idosos que são consideradas, na literatura, como fatores protetivos contra o DC. ${ }^{(15)} \mathrm{O}$ critério adotado para classificar o idoso como praticantes (sim) ou não praticantes (não) das práticas preventivas foi baseado nas recomendações de atividades em múltiplos domínios, por serem consideradas mais efetivas na prevenção do DC. ${ }^{(2)}$ Assim, foram considerados como praticantes os idosos que referiram realizar, no mínimo, três práticas, sendo uma de cada um destes domínios: (1) físico (atividade física, alimentação saudável, consumo moderado de bebida alcoólica e/ou não fumar); (2) social (visita a familiares ou amigos e/ou viajar, passear e/ou ter momentos de descontração); (3) mental (artesanato, leitura de livros ou jornais e/ou jogo de cartas, palavras cruzadas, dama, xadrez ou uso do computador).

Foi realizada análise descritiva dos dados do estudo. Posteriormente, foram dicotomizadas as variáveis independentes 'conhecimento e atitude', bem como a variável dependente 'práticas preventivas' para verificação de associação estatisticamente significativa por meio do teste $x^{2}$ de Pearson e análise de 
regressão múltipla de Poisson. Para a construção do modelo final, foram incluídas na análise aquelas variáveis que apresentaram $\mathrm{p}<0,20$ na análise univariada, utilizando para isso o método stepwise forward.

A pesquisa foi desenvolvida após aprovação do Comitê de Ética em Pesquisa do Hospital Universitário Júlio Müller, via Plataforma Brasil, sob parecer número 924.964/2014.

\section{RESULTADOS}

Dos 557 participantes do estudo, a maioria é do sexo feminino $344(61,8 \%)$; casada, $302(54,2 \%)$; aposentada, 397 (71,2\%); e recebe até um salário-mínimo, 392 (70,3\%). A maior parte, 283 (50,8\%), possui entre 60 e 69 anos; e é analfabeta, 235 (42,2\%). Quanto às condições de saúde, 513 (92,1\%) possui dois ou mais problemas de saúde, sendo os problemas cardiovasculares, $403(72,4 \%)$, e osteomusculares, $388(69,7 \%)$, mais frequentes. Quase a totalidade dos idosos, $464(83,3 \%)$, faz uso de medicamentos e a maior parte, 245 (43,9\%), autoavalia sua saúde como regular.

Sobre o conhecimento dos idosos em relação ao DC, $538(96,6 \%)$ já ouviu falar sobre a perda da memória nas pessoas mais velhas e sabe das consequências (553 - 99,3\% e 539 - 96,8\%, respectivamente). Além disso, quase todos os idosos $(91,6 \%)$ sabem que por ser idoso podem perder a memória. Porém, $389(70 \%)$ não sabem que o DC pode ser prevenido e desconhece as práticas para prevenção da perda da memória, tais como não ser obeso, 519 (93,2\%); jogar videogame e utilizar o computador, 488 $(87,6 \%)$; e viajar, passear ou ter momentos de descontração, 477 (85,6\%). (Tabela 1).

Tabela 1 - Distribuição dos idosos atendidos nas Unidades de Saúde da Família, segundo conhecimento sobre declínio cognitivo. Tangará da Serra, MT, Brasil, 2015

\begin{tabular}{|c|c|c|c|c|c|c|}
\hline \multirow{2}{*}{ Variáveis } & \multicolumn{2}{|c|}{ Sim } & \multicolumn{2}{|c|}{ Não } & \multicolumn{2}{|c|}{ Não sei } \\
\hline & $\mathbf{n}$ & $\%$ & $\mathbf{n}$ & $\%$ & $\mathbf{n}$ & $\%$ \\
\hline Ouviu falar sobre perda da memória nos idosos? & 538 & 96,6 & 19 & 03,4 & -- & -- \\
\hline Sabe que por ser idoso pode perder a memória? & 510 & 91,6 & -- & -- & 47 & 8,4 \\
\hline Sabe que perda da memória pode ser prevenida? & 168 & 30,2 & -- & -- & 389 & 69,8 \\
\hline Sabe que a perda da memória pode levar à necessidade de cuidador? & 553 & 99,3 & -- & -- & 4 & 00,7 \\
\hline Sabe que a perda da memória pode levar à internação e gastos em saúde? & 539 & 96,8 & -- & -- & 18 & 03,2 \\
\hline Jogos de videogame e utilizar o computador previnem a perda da memória? & 55 & 9,9 & 140 & 2,5 & 488 & 87,6 \\
\hline $\begin{array}{l}\text { Jogos de cartas, palavras cruzadas, dama ou xadrez previnem a perda da } \\
\text { memória? }\end{array}$ & 103 & 18,5 & 5 & 0,9 & 449 & 80,6 \\
\hline Ler livros ou jornais previne a perda da memória? & 142 & 25,5 & 9 & 01,6 & 406 & 72,9 \\
\hline Atividades de artesanato previnem a perda da memória? & 135 & 24,2 & 6 & 01,1 & 416 & 74,7 \\
\hline Viajar, passear ou ter momentos de descontração previne a perda da memória? & 56 & 10,1 & 24 & 04,3 & 477 & 85,6 \\
\hline Visitar e receber visitas de familiares e/ou amigos previne a perda da memória? & 70 & 12,6 & 19 & 03,4 & 468 & 84 \\
\hline Realizar atividade física previne a perda da memória? & 93 & 16,7 & 7 & 01,3 & 457 & 82 \\
\hline Não ser obeso previne a perda da memória? & 24 & 04,3 & 14 & 02,5 & 519 & 93,2 \\
\hline Alimentação saudável previne a perda da memória? & 169 & 30,3 & 3 & 00,5 & 385 & 69,2 \\
\hline Controlar doenças como pressão alta e diabetes previne a perda da memória? & 87 & 15,6 & 10 & 1,8 & 460 & 82,6 \\
\hline Consumir moderadamente bebida alcoólica previne a perda da memória? & 73 & 13,1 & 46 & 08,3 & 438 & 78,6 \\
\hline Não fumar previne a perda da memória? & 194 & 34,8 & 6 & 01,1 & 357 & 64,1 \\
\hline
\end{tabular}


Quanto às atitudes dos idosos em relação ao DC, 551 (98,9\%) acredita que a perda da memória é um problema sério; que podem perder a memória, 377 (67,7\%); e acham que podem depender de alguém, caso perca a memória, 545 (97,8\%). $553(63,4 \%)$ também se preocupam com a possibilidade de perder a memória; e em prevenir sua ocorrência, 402 (72,2\%). Quando questionados sobre o que acreditavam prevenir a perda da memória, houve predomínio de respostas de idosos que crêem que todas elas, com exceção do consumo moderado de álcool (25,9\%), previnem o DC. (Tabela 2).

Tabela 2 - Distribuição dos idosos atendidos nas Unidades de Saúde da Família, segundo atitudes sobre declínio cognitivo. Tangará da Serra, MT, Brasil, 2015

\begin{tabular}{|c|c|c|c|c|}
\hline \multirow{2}{*}{ Variáveis } & \multicolumn{2}{|c|}{ Sim } & \multicolumn{2}{|c|}{ Não } \\
\hline & $\mathbf{n}$ & $\%$ & $\mathbf{n}$ & $\%$ \\
\hline Acha que a perda da memória é um problema sério? & 551 & 98,9 & 6 & 01,1 \\
\hline Acha que pode perder a memória? & 377 & 67,7 & 180 & 32,3 \\
\hline Acha que pode depender de alguém caso perca a memória? & 545 & 97,8 & 12 & 02,2 \\
\hline Tem preocupação em perder a memória? & 353 & 63,4 & 204 & 36,6 \\
\hline Tem preocupação em prevenir a perda da memória? & 402 & 72,2 & 155 & 27,8 \\
\hline Acha que a perda da memória pode ser prevenida? & 450 & 80,8 & 107 & 19,2 \\
\hline Acha que deve procurar informações sobre prevenção? & 525 & 94,3 & 32 & 05,7 \\
\hline Acredita que jogos de videogame e utilizar o computador previnem a perda da memória? & 280 & 50,3 & 277 & 49,7 \\
\hline $\begin{array}{l}\text { Acredita que jogos de cartas, palavras cruzadas, dama ou xadrez previnem a perda da } \\
\text { memória? }\end{array}$ & 402 & 72,2 & 155 & 27,8 \\
\hline Acredita que ler livros ou jornais previne a perda da memória? & 471 & 84,6 & 86 & 15,4 \\
\hline Acredita que atividades de artesanato previnem a perda da memória? & 487 & 87,4 & 70 & 12,6 \\
\hline Acredita que viajar, passear ou ter momentos de descontração previne a perda da memória? & 482 & 86,5 & 75 & 13,5 \\
\hline Acredita que visitar e receber visitas de familiares e/ou amigos previne a perda da memória? & 488 & 87,6 & 69 & 12,4 \\
\hline Acredita que realizar atividade física previne a perda da memória? & 476 & 85,5 & 81 & 14,5 \\
\hline Acredita que não ser obeso previne a perda da memória? & 400 & 71,8 & 157 & 28,2 \\
\hline Acredita que alimentação saudável previne a perda da memória? & 524 & 94,1 & 33 & 05,9 \\
\hline Acredita que controlar doenças como pressão alta e diabetes previne a perda da memória? & 493 & 88,5 & 64 & 11,5 \\
\hline Acredita que consumir moderadamente bebida alcoólica previne a perda da memória? & 144 & 25,9 & 413 & 74,1 \\
\hline Acredita que não fumar previne a perda da memória? & 507 & 91 & 50 & 09 \\
\hline
\end{tabular}

As práticas preventivas de DC mais realizadas pelos idosos foram visitar familiares ou amigos, 445 (79,9\%); viajar, passear e/ou ter momentos de descontração, 343 (61,6\%); ler livros ou jornais, 239 (42,9\%); e atividade física, 199 (35,7\%).

A avaliação das variáveis do estudo por meio da prévia classificação mostrou que a maioria dos idosos possui conhecimento insatisfatório, 469 (84,2\%); atitude favorável, 523 (93,9\%); e realiza práticas preventivas, $307(55,1 \%)$. (Tabela 3). 
Tabela 3 - Distribuição dos idosos atendidos nas Unidades de Saúde da Família, segundo conhecimento, atitude e práticas preventivas de declínio cognitivo. Tangará da Serra, MT, Brasil, 2015

\begin{tabular}{|c|c|c|}
\hline Variáveis & Frequência (n) & Porcentagem (\%) \\
\hline \multicolumn{3}{|l|}{ Conhecimento } \\
\hline Satisfatório & 088 & 15,8 \\
\hline Insatisfatório & 469 & 84,2 \\
\hline \multicolumn{3}{|l|}{ Atitude } \\
\hline Favorável & 523 & 93,9 \\
\hline Não favorável & 034 & 6,1 \\
\hline \multicolumn{3}{|l|}{ Práticas preventivas } \\
\hline Sim & 307 & 55,1 \\
\hline Não & 250 & 44,9 \\
\hline TOTAL & 557 & 100 \\
\hline
\end{tabular}

A análise bivariada mostrou associação estatisticamente significativa entre conhecimento e práticas preventivas $(p=0,014)$. A regressão múltipla de Poisson foi realizada com as duas variáveis (conhecimento e atitude), a fim de atender o objetivo do estudo; e, após ajuste por idade, sexo e escolaridade, manteve-se a associação. A prevalência de práticas preventivas dos idosos foi maior (21\%) entre os que possuem conhecimento satisfatório $(\mathrm{RP}=1,21 ; \mathrm{IC}=1,02-1,43)$ quando comparados com aqueles que têm conhecimento insatisfatório, independente da atitude, idade, sexo e escolaridade. (Tabela 4).

Tabela 4 - Modelo de regressão múltipla de Poisson: variáveis associadas às práticas preventivas de declínio cognitivo entre os idosos atendidos nas Unidades de Saúde da Família. Tangará da Serra, MT, Brasil, 2015

\begin{tabular}{lcccc}
\hline $\begin{array}{l}\text { Variáveis } \\
\text { Conhecimento } \\
\text { Satisfatório }\end{array}$ & Prevalência (\%) & RP bruta (IC95\%)+ & RP Ajustada (IC95\%) $)$ & Valor de $\mathbf{p}$ \\
Insatisfatório & 67 & $1,26(1,06-1,50)$ & $1,21(1,02-1,43)$ & $\mathbf{0 , 0 2 7}$ \\
$\begin{array}{l}\text { Atitude } \\
\text { Favorável }\end{array}$ & 52,9 & 1 & 1 & \\
Não favorável & 55,6 & $1,18(0,82-1,70)$ & $1,14(0,79-1,64)$ & 0,459 \\
\hline
\end{tabular}

tRP: Razão de prevalência; IC: intervalo de confiança. ‡Ajustado por idade, sexo e escolaridade.

\section{DISCUSSÃO}

Este é o primeiro estudo que se tem conhecimento que explora o conhecimento, as atitudes e as práticas de idosos sobre o DC conjuntamente. Outros estudos pesquisaram separadamente esses fatores. $(10,12,17)$ Além disso, é o primeiro que estudou a associação entre essas variáveis.

Embora a maioria dos participantes possua compreensão geral sobre o DC, seu conhecimento sobre prevenção é insatisfatório. Eles desconhecem que o DC pode ser prevenido e que as práticas preventivas recomendadas podem retardar seu surgimento ou evitá-lo. Provavelmente, esse achado possa ser atribuído à baixa renda e escolaridade da população estudada, características geralmente associadas ao baixo conhecimento. Isso indica que idosos ainda necessitam de mais informações sobre o DC. O saber contribui para que as pessoas tomem consciência dos seus problemas de saúde e adotem medidas de prevenção. ${ }^{(18)}$ 
Um dos achados relevantes desta pesquisa foi a preocupação dos participantes com o DC e sua prevenção, e está em concordância com estudos realizados tanto com idosos quanto com pessoas mais jovens. ${ }^{(12,17)}$

Outro resultado encontrado nesta pesquisa foi que a maioria dos participantes acredita que as práticas preventivas de DC o previnem. Muitas pessoas acreditam que manter-se mentalmente ativo, ter uma dieta saudável e realizar atividade física ajuda a prevenir a DA. ${ }^{(11)}$ Esse achado é importante, pois há pessoas que acreditam que nada pode ser feito para prevenir o DC. ${ }^{(19)}$ Provavelmente, os idosos deste estudo tenham atitudes favoráveis às práticas preventivas de DC porque se percebem vulneráveis à condição. A percepção de vulnerabilidade à doença e da probabilidade de desenvolvê-la é um fator que pode influenciar nos comportamentos preventivos de saúde. ${ }^{(12)}$

Neste estudo, embora a maioria dos participantes da pesquisa tenha conhecimento insatisfatório sobre as práticas preventivas de DC, suas atitudes em relação a elas são favoráveis. Essa divergência mostra que as atitudes nem sempre dependem do conhecimento que as pessoas têm sobre determinado tema. Elas são construídas ao longo da vida da pessoa e sofrem influência do conhecimento, mas igualmente da cultura, das interações e experiências. ${ }^{(20)}$ Isso também mostra que as atitudes devem ser um fator a ser considerado e trabalhado nas ações de prevenção dos profissionais de saúde quando se relacionam com os idosos.

Nesta investigação, é surpreendente que muitos dos idosos realizem as práticas preventivas de DC apresentadas a eles e de forma espontânea. Os estudos de prática preventiva de DC encontrados são de intervenções que avaliam os efeitos de programas de prevenção de DC na função cognitiva dos idosos ${ }^{(21-22)}$ e, geralmente, a adesão das pessoas a práticas de prevenção é maior em programas supervisionados. ${ }^{(23)}$ Provavelmente, os idosos pesquisados praticam as atividades preventivas porque possuem atitudes favoráveis a elas. Mesmo não sabendo que elas previnem o DC, os idosos acreditam que podem, de alguma forma, beneficiar-se delas.

A associação entre as práticas preventivas de DC e o conhecimento foi o principal achado deste estudo. Mesmo que a proporção de idosos com conhecimento insatisfatório tenha sido maior, a prevalência de práticas preventivas foi maior nos idosos que possuem conhecimento satisfatório. Como mencionado anteriormente, pesquisas de conhecimento, atitudes e práticas de idosos relacionadas ao DC não foram encontradas na literatura. Portanto, a comparação desse achado com outros estudos não é possível.

As características da população estudada podem ser uma provável explicação para essa associação. A maioria dos participantes é do sexo feminino. Pode ser que, pelo fato de as mulheres procurarem mais os serviços de saúde e realizarem práticas preventivas, ${ }^{(20)}$ elas tenham mais acesso às informações sobre os problemas de saúde. Do mesmo modo, a faixa etária da maior parte da população estudada é de idosos jovens. Supõe-se que, por serem mais jovens, têm mais contato com informações fornecidas por meios de comunicação e tecnologias. Maior conhecimento sobre o DC pode ser atribuído ao aumento da divulgação da doença na mídia, assim como aos crescentes esforços de instituições governamentais e não governamentais para ampliar o conhecimento dessa condição e sua prevenção. ${ }^{(12)}$

Este estudo tem como limitação o fato de não existir instrumentos validados que permitam a avaliação do objeto de investigação e os dados terem sido obtidos por meio de autorrelato; entretanto, este método pode ser considerado apropriado para situações particulares, ${ }^{(24)}$ além de ser discreto e capaz de distinguir entre adesão intencional e não intencional e comportamentos. ${ }^{(25)}$

\section{CONCLUSÃO}

Neste estudo, a maioria dos idosos possui conhecimento insatisfatório, atitudes favoráveis sobre a condição e realiza práticas preventivas de DC. A prevalência de práticas preventivas foi maior nos que possuem conhecimento satisfatório. Isso provavelmente se deve às características da população estudada que teria mais conhecimento do DC provindo da busca e acesso às informações sobre essa condição. 
Os resultados contribuem para a compreensão do conhecimento e atitudes que os idosos têm sobre o DC, e isso pode ser usado no planejamento de intervenções pelos profissionais de saúde, principalmente os enfermeiros, como, também, para realizar educação em saúde sobre as causas e consequências, bem como comportamentos que auxiliam na prevenção do declínio cognitivo para a população em geral. Além disso, podem subsidiar os governantes na adoção de políticas públicas de envelhecimento saudável.

Tais achados também reforçam a necessidade de que os profissionais de saúde da atenção básica, em especial os enfermeiros, desenvolvam ações de promoção à saúde da população que envelhece, por meio de programas de prevenção do DC que incluam as práticas preventivas, já evidenciadas pela ciência, como contribuintes da saúde cognitiva.

\section{REFERÊNCIAS}

1. Organización Mundial de la Salud (WHO). Organización Pan americana de La Salud.Demencia: una prioridad de salud pública. [Internet] Washington: WHO; 2013 [acesso em 05 ago 2015]. Disponível: http://apps.who.int/iris/ bitstream/10665/98377/1/9789275318256_spa.pdf.

2. Alzheimer's Disease International (ADI). World Alzheimer Report 2014. Dementia and Risk Reduction - an analysis of protective and modifiable factors. [Internet] London: ADI; 2014 [acesso em 30 nov 2014]. Disponível: https://www. alz.co.uk/research/WorldAlzheimerReport2014.pdf.

3. Alzheimer'sDiseaselnternational (ADI). Bupa. Relatório ADI/Bupa, Demência nas Américas: Custo atual e futuro e prevalência da doença de Alzheimer e outras demências. [Internet] London: ADI; 2013. [acesso em 23 jul 2015]. Disponível: https://www.alz.co.uk/sites/default/files/pdfs/dementia-in-the-americas-BRAZILIAN-PORTUGUESE.pdf.

4. Charchat-Fichman H, Caramelli P, Sameshima K, Nitrini R. Declínio da capacidade cognitiva durante o envelhecimento. Rev. Bras. Psiquiatr. [Internet] 2005;27(12) [acesso em 12 fev 2015]. Disponível: http://dx.doi.org/10.1590/S151644462005000100017.

5. Netto MP. Tratado de Gerontologia. 2ed. São Paulo: Atheneu; 2007.1750 p.

6. Imtiaz B, Tolppanen A, Kivipelto M, Soininen H. Future directions in Alzheimer's disease from risk factors to prevention. BiochemPharmacol. [Internet] 2014;88(4) [acesso em 08 fev2015]. Disponível: https://doi.org/10.1016/j. bcp.2014.01.003.

7. Tak ECPM, Uffelen JGZ, Paw MJMCA, Mechelen W, Hopman-Rock M. Adherence to exercise programs and determinants of maintenance in older adults with mild cognitive impairment. J AgingPhysAct. [Internet] 2012;20(1) [acesso em 17 dez 2014]. Disponível: https://research.vu.nl/ws/portalfiles/portal/727971.

8. Garmendia ML, Dangour AD, Albala C, Eguiguren P, Allen E, Uauy R. Adherence to a physical activity intervention among older adults in a post-transitional middle income country: a quantitative and qualitative analysis. J Nutr Health Aging. [Internet] 2013;17(5) [acesso em 11 jul 2015]. Disponível: http://dx.doi.org/10.1007/s12603-012-0417-1.

9. Lam LCW, Cheng ST. Maintaining long-term adherence to lifestyle interventions for cognitive health in late life. IntPsychogeriatr. [Internet] 2013;25(2) [acesso em 10 dez 2014]. Disponível: https://doi.org/10.1017/ S1041610212001603.

10. Sun F, Gao X, Shen H, Burnette D. Levels and correlates of knowledge about alzheimer's disease among older chineseamericans. J Cross Cult Gerontol. [Internet] 2014;29(2):173-83. [acesso em 28 nov]. Disponível: http://dx.doi. org/10.1007/s10823-014-9229-6.

11. Ayalon L. Re-examining ethnic differences in concerns, knowledge, and beliefs about Alzheimer's disease: results from a national sample. Int J GeriatrPsychiatry. [Internet] 2013;28(12) [acessoem 31 out 2014]. Disponível: http://dx.doi. org/10.1002/gps.3959.

12. Werner P, Goldberg S, Mandel S, Korczyn AD. Gender differences in lay persons' beliefs and knowledge about Alzheimer's disease (AD): A national representative study of Israeli adults. Arch GerontolGeriatr. [Internet] 2013;56(2) [acessoem 11 dez 2014]. Disponível: http://dx.doi.org/10.1016/j.archger.2012.11.001. 
13. Ministério da Saúde (BR). Envelhecimento e saúde da pessoa idosa. Secretaria de Atenção à Saúde, Departamento de Atenção Básica. [Internet] Brasília: Ministério da Saúde; 2007 [acesso em 23 out 2014]. Disponível: http://bvsms. saude.gov.br/bvs/publicacoes/evelhecimento_saude_pessoa_idosa.pdf.

14. World Health Organization (WHO). Advocacy, communication and social mobilization for TB control: a guide to developing knowledge, attitude and practice survey. [Internet] Geneva: WHO; 2008. [acesso em 02 jun 2014 ]. Disponível: http://apps.who.int/iris/bitstream/10665/43790/1/9789241596176_eng.pdf.

15. Kaliyaperumal K. Guideline for Conducting a Knowledge, Attitude and Practice (KAP) Study. AECS Illumination. [Internet] 2004;4(1) [acesso em 28 jun 2014]. Disponível: http://v2020eresource.org/content/files/guideline_kap_Jan_ mar04.pdf.

16. Marinho LAB, Costa-Gurgel MS, Cecatti JG, Osis MJD. Conhecimento, atitude e prática do autoexame das mamas em centros de saúde. Rev Saúde Pública. [Internet] 2003;37(5) [acesso em 05 jul 2014]. Disponível: http://dx.doi. org/10.1590/S0034-89102003000500005.

17. Rovner BW, Casten RJ, Harris LF. Cultural Diversity and Views on Alzheimer Disease in Older African Americans. Alzheimer Dis Assoc. Disord. [Internet] 2013;27(2) [acesso em 15 nov 2014]. Dísponível: http://dx.doi.org/10.1097/ WAD.0b013e3182654794.

18. Ministério da Saúde (BR). Pesquisa de conhecimento, atitudes e práticas na população brasileira.Secretaria de Vigilância em Saúde. Departamento de DST, Aids e Hepatites Virais. [Internet] Brasília: Ministério da Saúde; 2011 [acesso em 02 nov 2018]. Disponível: http://bvsms.saude.gov.br/bvs/publicacoes/pesquisa_conhecimentos_atitudes_ praticas_populacao_brasileira.pdf.

19. Anderson LN, McCaulb KD, Langley LK. Common-sense beliefs about the prevention of Alzheimer's disease. AgingMent Health. [Internet] 2011;15(7) [acesso em 12 dez 2014]. Disponível: http://dx.doi.org/10.1080/13607863.2 011.569478 .

20. Brito AMM, Camargo BV. Representações sociais, crenças e comportamentos de saúde: um estudo comparativo entre homens e mulheres. Temas Psicol. [Internet] 2011;19(1) [acesso em 11 set 2015]. Disponível: http://pepsic. bvsalud.org/pdf/tp/v19n1/v19n1a23.pdf.

21. Cox KL, Flicker L, Almeida OP, Xiao J, Greenop KR, Hendriks J, et al. The FABS trial: A randomised control trial of the effects of a 6-month physical activity intervention on adherence and long-term physical activity and self-efficacy in older adults with memory complaints. Prev Med. [Internet] 2013;57(6) [acesso em 18 ago 2015]. Disponível: http:// dx.doi.org/10.1016/j.ypmed.2013.09.010.

22. Rebok GW, Ball K, Guey LT, Jones RN, Kim H, King JW, et al. Ten-year effects of the advanced cognitive training for independent and vital elderly cognitive training trial on cognition and everyday functioning in older adults. J AmGeriatr Soc. [Internet] 2014;62(1) [acesso em 15 jul 2015]. Disponível: http://dx.doi.org/10.1111/jgs.12607.

23. Picorelli AMA, Pereira LSM, Pereira DS, Felício D, Sherrington C. Adherence to exercise programs for older people is influenced by program characteristics and personal factors: a systematic review. J Physiother. [Internet] 2014;60(3) [acesso em 30 jul 2015]. Disponível: http://dx.doi.org/10.1016/j.jphys.2014.06.012.

24. Conway JM. Lance CE. Whatreviewers should expect from authors regarding common method bias in organizational research. J Bus Psychol. [Internet] 2010;25(3) [acesso em 13 nov 2015]. Disponível: http://dx.doi.org/10.1007/s10869010-9181-6.

25. Garfield S, Clifford S, Eliasson L, Barber N, Willson A. Suitability of measures of self-reported medication adherence for routine clinical use: A systematic review. BMC Med Res Methodol. [Internet] 2011;11(149) [acesso em 10 nov 2015]. Disponível: https://doi.org/10.1186/1471-2288-11-149. 